\title{
Los síndromes ansiosos y depresivos como timopatías
}

Otto Dörr Zegers*

Resumen: En otro estudio intentamos demostrar la conveniencia de conceptualizar las esquizofrenias como "logopatías". En esta oportunidad se trata de demostrar que todas las enfermedades del ánimo y gran parte de los llamados trastornos de ansiedad pueden ser concebidos como una sola forma del enfermar humano, cual es la perturbación de la disposición afectiva, en el sentido de Heidegger. Basamos esta hipótesis en tres hechos de observación: $1^{\mathrm{o}}$ Investigaciones recientes no han permitido demostrar la consistencia de constructos como "depresión mayor" y "trastorno de ansiedad generalizada". Más aún, estudios evolutivos han señalado más bien que estas supuestas entidades nosológicas - definidas con criterios operacionales - se sustituyen en el mismo paciente a lo largo de la vida. Por otra parte, López-Ibor Sr. (1950), sobre la base de lo sostenido por algunos autores clásicos y sus propias observaciones, propuso emplear para todos estos cuadros un término genérico, "timopatía", con una vertiente ansiosa y otra depresiva, idea que compartimos plenamente y a la que intentamos dar una fundamentación evolutiva y filosófica. $2^{\circ}$ Hay elementos que permiten sospechar que estas enfermedades son connaturales al ser humano y que es probable que - al igual que la esquizofrenia - hayan aparecido junto con el cambio evolutivo que permitió al homo erectus transformarse en homo sapiens. Hay por lo menos dos hechos que apoyan esta hipótesis. Uno es que las primeras palabras expresadas por el hombre tienen en común la raíz aj, ankh, ajo, agu, todas las cuales representan el origen de la palabra angustia, síntoma fundamental tanto de los cuadros ansiosos como de los depresivos. El otro tiene que ver con la creatividad, que es definitorio de lo humano y que las más diversas investigaciones vinculan con la más importante de las enfermedades del ánimo, la forma bipolar. $3^{\circ}$ En su

\footnotetext{
* Profesor de Psiquiatría de la Universidad de Chile y de la Universidad Diego Portales. E-mail: odoerrz@gmail.com.
}

Psicopatologia Fenomenológica Contemporânea, 2014, 3 (1), 1-22 


\section{Los síndromes ansiosos y depresivos como timopatías}

descripción fenomenológica del Dasein en Ser y Tiempo, Heidegger afirma que el hombre está en el mundo como comprensión (lenguaje) y como disposición afectiva. No deja de ser llamativo el que estas dos formas esenciales de enfermar el ser humano correspondan justamente a estos dos modos fundamentales de ser en el mundo y por ende, nos parece también filosóficamente justificada la polaridad logopatías - timopatías.

Palabras-llave: Ansiedad; depresión, timopatía.

Abstract: In another study we tried to demonstrate the convenience of conceptualizing schizophrenias as "logopathies". In this study we try to demonstrate that all mood disorders and the majority of the anxiety disorders can be understood as a sole form of human disease, related to the Heideggerain notion of Befindlichkeit. They are a trouble of the Befindlichkeit. We based this hypothesis on three facts: $1^{\circ}$. There is no consistency in such terms as "major depression" and "generalized anxiety disorder". Lopez-Ibor Sr. (1950) proposed the term "timopathy" to describe them; $2^{\circ}$. There are elements to believe that these diseases are connatural to the human being and it is likely that they appeared as an evolutionary movement leading from homo erectus to homo sapiens; $3^{\circ}$ In his phenomenological descriptionof the Dasein in Being and Time, Heidegger states that the human being is in the world as comprehension (language) and Befindlichkeit. These two forms of being troubled correspond to these two fundamental modes of being in the world.

Keywords: Anxiety, depression; timopathy.

En otro trabajo (2010), intentamos demostrar la conveniencia de conceptualizar las esquizofrenias y enfermedades afines como "logopatías". Fundamentamos nuestra hipótesis en tres hechos de observación. Primero, que la experiencia clínica y las descripciones de los más importantes autores clásicos

Psicopatologia Fenomenológica Contemporânea, 2014, 3 (1), 1-22 


\section{Otto Dörr Zegers}

y de algunos modernos permiten concluir que el fenómeno central de la esquizofrenia es la alteración del pensamiento y/o lenguaje. Todo el resto de los síntomas, incluidos los llamados síntomas negativos, serían inespecíficos. Segundo, que investigaciones recientes sugieren que la esquizofrenia es un elemento constitutivo de la naturaleza humana y la mutación que le dio origen es contemporánea de aquella que permitió al homo erectus adquirir el lenguaje abstracto y con ello su condición de homo sapiens. Tercero, que en la revolucionaria concepción de Martin Heidegger del ser humano como Dasein, se describen tres características fundamentales de su modo de estar en el mundo: la comprensibilidad, el lenguaje y la disposición afectiva o disposicionalidad (Befindlichkeit). Como las dos primeras pueden tomarse en cierto modo como una sola, puesto que no hay lenguaje sin comprensibilidad ni comprensibilidad sin lenguaje, bien podríamos hablar de una polaridad entre comprensibilidad (lenguaje) y disposicionalidad (encontrarse, según otras versiones), constituyendo las esquizofrenias un claro ejemplo de la perturbación de la primera de ellas. Ahora trataremos de demostrar que todas las enfermedades del ánimo y gran parte de los llamados trastornos de ansiedad sobre todo aquellos cuya sintomatología no sólo es expresión de un trastorno de personalidad - podrían ser concebidas como una sola forma del enfermar humano, cual es la perturbación de la disposicionalidad en el sentido de Heidegger.

\section{El problema de la definición y clasificación de los trastornos del ánimo}

La depresión ha sido definida en distintas formas desde el comienzo de la psiquiatría científica, con Kraepelin (1996), hasta el día de hoy. Si uno quisiera establecer una línea diferencial entre todas las formas de concebir la depresión, esta tendría que ser la pretensión o no de determinar dentro de la multiplicidad de sus manifestaciones, algunos síntomas fundamentales o necesarios. Ejemplos de lo segundo, vale decir, de aquellos que no aspiran a 


\section{Los síndromes ansiosos y depresivos como timopatías}

establecer la existencia de síntomas básicos o fundamentales, son los modernos sistemas de clasificación y diagnóstico norteamericanos, desde los criterios de la escuela de Saint Louis (1972) hasta el DSM-IV-TR (2000), y en menor medida el ICD 10. Fieles al método categorial que pretenden aplicar, estos sistemas hacen una lista de los síntomas más diversos y exigen la presencia de tres, cuatro o cinco de ellos para afirmar o negar la existencia de un "trastorno depresivo". La relación entre estos síntomas es simplemente de contigüidad. Así, en los criterios de Saint Louis (8 más la perturbación del ánimo) aparece la pérdida de la energía al lado de la perturbación del sueño y de los auto-reproches. Los Research Diagnostic Criteria (RDC) (1982) también exigen la presencia de cinco de ocho síntomas, que resultan ser los mismos que los de la escuela de Saint Louis.

Entre los autores clásicos, en cambio, ha predominado siempre la idea de buscar síntomas fundamentales y separarlos de los accesorios. Así, por ejemplo, Kraepelin, en su Introducción a la Psiquiatría (1916), postula que los síntomas fundamentales son la distimia depresiva o ansiosa, la dificultad para pensar y la inhibición de la voluntad. Eugen Bleuler, en la primera edición de su Tratado de Psiquiatría del mismo año del libro de Kraepelin que estamos citando (1916), y sin cambios sustanciales con respecto a las ediciones hechas a lo largo del siglo XX por su hijo Manfred, también plantea que hay tres síntomas fundamentales, que él llama "cardinales" y que prácticamente se superponen con los de Kraepelin: distimia depresiva, inhibición del curso del pensar e inhibición de lo que él llama las "funciones centrífugas" (atender, concentrarse, memorizar, tomar decisiones). Se puede observar que aquí la "inhibición de las funciones centrífugas" (esas que nos conectan con el medio ambiente) reemplaza a la "inhibición de la voluntad" de Kraepelin, pero no cabe duda que ambos autores están aludiendo a lo mismo, eso que más tarde Binswanger (1960) llamará “das Nicht-Können", el no-poder. Kurt Schneider también creyó encontrar la perturbación fundamental de la depresión. Siguiendo la fenomenología de los sentimientos de Max Scheler, Schneider concibió las enfermedades del ánimo 


\section{Otto Dörr Zegers}

como alteraciones de un tipo particular de sentimientos, los vitales, caracterizados por su proximidad al cuerpo y su carácter difuso, y dentro de ellos describió como particularmente específicos los "síntomas vitales" que hoy llevan su nombre (opresión precordial, globus melancholicus y nucalgia). En 1969 otro autor alemán, W. M. Pfeiffer, publicó los resultados de un estudio transcultural realizado en Alemania e Indonesia, concluyendo que los síntomas fundamentales de esta enfermedad, vale decir, aquellos que están siempre presentes y no son determinados por el tipo o grado de civilización o cultura, eran sólo tres: un compromiso del ánimo "hacia el polo depresivo" y "difícil de definir", la presencia de sensaciones corporales anormales y la alteración de funciones vegetativas, como el sueño y el apetito.

A partir de 1971 este autor $(1979,1980,1993)$ ha realizado una serie de estudios tanto empíricos como fenomenológicos orientados también a definir, como los autores clásicos, los fenómenos fundamentales de la depresión. El autor concluyó que lo común a todos los cuadros depresivos propiamente tales eran tres fenómenos muy específicos, a saber:

\section{Fenómeno del cambio del cuerpo vivido o corporalidad:}

Aquí pertenecen todos aquellos síntomas que surgen como manifestaciones de un modo distinto del estar el depresivo en su cuerpo (Befinden). Desde la perspectiva del paciente, estos síntomas son: decaimiento, falta de ánimo y de fuerzas, sensación de pesadez corporal, sensación de frío, sensación de náuseas, angustia, más los clásicos síntomas vitales como la opresión precordial, el globus melancholicus, la sensación de presión dolorosa en la nuca. El grado extremo de este cambio de la corporalidad sería el síndrome de Cotard, con la negación de órganos. Desde la perspectiva del observador, este cambio es visto como palidez, arruga frontal en omega, ceño fruncido, opacidad de la mirada, párpados de Veraguth, expresión de angustia congelada. Este conjunto de síntomas configuran un fenómeno que afecta globalmente a la

Psicopatologia Fenomenológica Contemporânea, 2014, 3 (1), 1-22 


\section{Los síndromes ansiosos y depresivos como timopatías}

corporalidad del depresivo y que este autor ha descrito como "cadaverización" (Dörr, 1979, 1980), siendo su versión más extrema el estado de estupor.

2. Fenómeno de la alteración del cuerpo en acción u operante:

Desde la perspectiva del paciente, el fenómeno de la inhibición se presenta como un deterioro de todas las funciones que Bleuler llamara "centrífugas", vale decir, aquellas que nos conectan con el mundo externo: dificultad para pensar, concentrarse, decidirse, realizar las tareas habituales, pérdida de las capacidades de atención y de memoria, etc. (fenómeno del "nopoder" de Binswanger, 1960). Desde la perspectiva del observador, la inhibición es idéntica a un enlentecimiento general, el cual se manifiesta concretamente como lentitud en los movimientos, tendencia a permanecer estático, latencia en las respuestas, hablar dificultoso y pérdida de fuerza de la voz.

\section{Fenómeno de la alteración de la ritmicidad biológica:}

Todos los ritmos biológicos se encuentran alterados, invertidos o suspendidos: se altera el ritmo sueño-vigilia, se invierte el ritmo circadiano, se suspende el ciclo menstrual, etc. Disminuye el apetito y la libido, el ritmo digestivo se hace más lento o más rápido; lo mismo ocurre con el ritmo urinario. Este compromiso de la ritmicidad biológica va empero más allá de la corporalidad de cada sujeto, en el sentido que los síntomas o la enfermedad misma se presentan ligados a los ciclos cósmicos: periodicidad diaria, semanal, mensual, estacional y de la vida entera.

Algunos años más tarde, un grupo de investigadores de la Universidad de Viena dirigidos por el Prof. Peter Berner (1983) describió el "síndrome ciclotímico axial endomorfo", válido para todas las enfermedades del ánimo, con variaciones en la tonalidad afectiva, por cierto, según se trate de una depresión pura, de una manía o de un cuadro mixto, consistente en dos 


\section{Otto Dörr Zegers}

fenómenos fundamentales: el compromiso del cuerpo vivido, disposición afectiva o disposicionalidad (Befindlichkeit) y la perturbación de los ritmos vitales. Es llamativa la coincidencia con los resultados de nuestras investigaciones. La diferencia se limita al hecho que Berner considera como un solo fenómeno la alteración del cuerpo vivido y la del cuerpo operante.

En todo caso, ni los criterios de Viena ni los nuestros han tenido una mayor repercusión y la psiquiatría internacional se rige hasta el día de hoy y desde al menos 1980, con la aparición del DSM III, por criterios categoriales. Pero ocurre que en los últimos años han empezado a aparecer en la literatura una serie de críticas a la aplicación de estos sistemas en la psiquiatría en general y en el campo de las depresiones en particular. Tempranamente Anne Farmer y Peter McGuffin (1989) plantearon dudas sobre la viabilidad de la aplicación de estos criterios. Sus críticas pueden resumirse como sigue: 1. Estos sistemas dependen principalmente de los síntomas subjetivos manifestados por los pacientes y toman poco en cuenta la información de terceros. 2. Se establece una distinción demasiado clara entre el trastorno (Eje I) y la personalidad (Eje II), lo que resulta arbitrario desde el punto de vista clínico. 3. Se ignora el contexto sociocultural del paciente. 4. Olvidando las complejidades de los cuadros clínico-psiquiátricos y sus muchos matices y transiciones, el clínico, al emplear los sistemas de diagnóstico operacional, tiene que decidir cada vez si un caso particular "cumple o no cumple con los criterios" para un determinado diagnóstico, en este caso una depresión. Casi dos décadas más tarde Gordon Parker (2005) realiza una revisión crítica del concepto de depresión mayor, concluyendo que este fracasa en cumplir los criterios ortodoxos de validez, puesto que sus límites son poco claros, no predice el curso natural en los casos concretos, ni está asociado tampoco a un tratamiento específico. Este autor piensa también que la abstinencia de toda consideración etiológica en el concepto trae más inconvenientes que ventajas, por cuanto la experiencia clínica demuestra que múltiples factores psicológicos, sociales y biológicos pueden contribuir a la génesis de una depresión. Un año más tarde el mismo Parker 


\section{Los síndromes ansiosos y depresivos como timopatías}

(2006) declara definitivamente la "inutilidad" del concepto de depresión mayor de los DSM. Fuera de las críticas planteadas en 2005, en este trabajo Parker va más al detalle y muestra de qué manera una misma manifestación, como los sentimientos de culpa y de minusvalía, por ejemplo, es considerada criterio diagnóstico para tres categorías diferentes: el episodio depresivo mayor, el episodio depresivo mayor de tipo melancólico y el con elementos psicóticos. Pero la crítica más dura a las actuales conceptualizaciones de los cuadros depresivos fue publicada recientemente en el British Journal of Psychiatry por James Cole y colaboradores (2008). Este autor habla al respecto de una "historia de confusiones" y más allá del caso específico de los cuadros depresivos, plantea serias dudas sobre la posibilidad de emplear en psiquiatría los criterios categoriales. Sus argumentos son primero, que al emplear definiciones operacionales, aquellos individuos que no cumplen con los criterios son clasificados en la categoría "no especificada de otra manera" (not otherwise specified). En segundo lugar, que las definiciones del trastorno son tan amplias e inestables que su confiabilidad es muy baja. Y por último, al estar orientadas principalmente a los síntomas más positivos, dejan de lado una cantidad de experiencias del paciente, las que podrían ser importantes tanto para su comprensión como para su tratamiento.

El cambiar los criterios categoriales por sistemas dimensionales tampoco sirve de mucho, por cuanto estos últimos de algún modo suponen los anteriores. Así, se define una categoría y se determinan luego arbitrariamente los grados de intensidad en la forma de presentación de esa categoría y, en el mejor de los casos, se sugiere la existencia de transiciones entre subtipos, como por ejemplo entre la forma bipolar y la monopolar de depresión. Una combinación de criterios categoriales y dimensionales es lo que propone uno de los más severos críticos de los sistemas categoriales en el diagnóstico y clasificación de las enfermedades del ánimo, el mismo James Cole (2008). Él termina concluyendo, sin embargo, que mientras no conozcamos la etiología de estos trastornos, será 


\section{Otto Dörr Zegers}

difícil una comprensión en profundidad y una "verdadera" clasificación de ellos.

La psiquiatría fenomenológica ha obviado el problema aplicando, desde Karl Jaspers (1959), el diagnóstico tipológico. Los trastornos mentales sin fundamento orgánico no serían "entes en sí”, sino tipos, vale decir, configuraciones o formas de darse la realidad psicopatológica a las que no se puede acceder ni por métodos cuantitativos, ni por consensos, sino sólo por medio de la intuición fenomenológica. Se habla aquí de tipos ideales, porque el caso particular nunca corresponde exactamente a esa idealidad. Cada caso concreto se aproxima más o menos a ese ideal que hemos aprendido a conocer junto a un maestro, sea éste una esquizofrenia, una enfermedad bipolar, un determinado trastorno de personalidad, etc. (Schwartz y Wiggins, 1985, 1987). Este método permite dar razón de las múltiples formas de presentación de los cuadros psiquiátricos y de sus transiciones tanto hacia otros tipos ideales como hacia la normalidad. La teoría de los tipos ideales, que Karl Jaspers tomó originalmente del famoso sociólogo y filósofo Max Weber, permite también comprender y eventualmente clasificar las polaridades que se dan comúnmente en ese ámbito de la realidad que es la psicopatología. Un ejemplo sería la polaridad entre el mundo de la esquizofrenia y el mundo de la psicosis maníaco-depresiva. Otra, ya dentro de las enfermedades del ánimo, entre la monopolaridad y la bipolaridad. Un tercer ejemplo muy iluminador es la polaridad entre la personalidad histérica y la personalidad obsesiva, etc. En suma, la aplicación en psiquiatría de los tipos ideales permite superar casi todos los inconvenientes que conllevan los sistemas categoriales e incluye, por definición, la dimensionalidad. Por último, ellos abren el camino hacia una psicopatología dialéctica que será, en nuestra opinión, la psicopatología del futuro y la más acorde con los nuevos desarrollos de la filosofía y de las ciencias.

Aunque no es este el tema específico al que nos queremos abocar en el día de hoy, nos pareció importante dibujar el marco dentro del cual nuestra 


\section{Los síndromes ansiosos y depresivos como timopatías}

propuesta de concebir los trastornos del ánimo como timopatías podría tener un sentido.

\section{El problema de la definición y clasificación de los trastornos ansiosos}

Lo que hoy los modernos sistemas de clasificación y diagnóstico consideran como trastornos ansiosos constituyen una arbitraria atribución a una sola categoría de los síndromes psicopatológicos más dispares. Uno, el trastorno de pánico, está basado en la existencia de un síntoma en particular, cual es la crisis de pánico con o sin agorafobia. La fobia social corresponde más a un trastorno de personalidad (timidez, sensitividad) que a un trastorno del eje I. El trastorno obsesivo-compulsivo es una enfermedad muy compleja, vinculada por un lado a la herencia y por otro a una muy probable base orgánica. El llamado stress post traumático es también un cuadro de mucha complejidad y que fue estudiado con detención por von Baeyer y colaboradores (1964) a propósito de los perseguidos por el régimen nazi. Los casos son muy distintos unos de otros según sea la situación desencadenante. Es muy diferente haber vivido una situación puntual, como un terremoto, y quedar algunos días o semanas con insomnio y expectación ansiosa, que haber sido discriminado, perseguido y torturado. Esta última situación, como lo describiera en forma impresionante von Baeyer, puede provocar un cambio de personalidad muy próximo a un desarrollo paranoídeo y en todo caso un cuadro que en su esencia nada tiene que ver con los cuadros post traumáticos por desastres naturales. Para hacer aún más caótica la categoría el DSM IV TR incluye en ella cuadros ansiosos provocados por sustancias (alcohol, anfetaminas, marihuana, etc.) y los "no clasificados de otra manera". Estos últimos permiten la entrada a la categoría de casos anecdóticos, en los que se combinan los más diversos rasgos de personalidad y de situaciones supuestamente traumáticas.

Mucho más acorde con la realidad clínica nos parecen los constructos empleados desde la época de Freud, sobre todo el de "neurosis de angustia". Y 


\section{Otto Dörr Zegers}

esto a pesar de su contaminación con la teoría psicoanalítica. Ese tipo ideal incluye una sintomatología que abarca lo que hoy se llama trastorno generalizado de ansiedad y el trastorno de pánico, con y sin agorafobia, pero también una personalidad determinada, con rasgos fuertemente dependientes, así como elementos biográficos más o menos característicos, a los que no podemos referirnos ahora. Tampoco corresponde discutir los mecanismos psicodinámicos que subyacerían a este cuadro. Sólo queremos señalar la mayor consistencia de este constructo sin pretensiones categoriales y que fuera abandonado hace ya décadas, en gran parte por el afán de los DSM de prescindir en sus descripciones de cualquier forma de consideración etiopatogénica.

Pero entre el concepto psicoanalítico de "neurosis de angustia" y el norteamericano de "trastorno de ansiedad" hubo un autor que planteó algunas ideas muy novedosas y fundamentales para la comprensión de estos cuadros. Por desgracia estos aportes pasaron prácticamente desapercibidos, por lo menos en el ámbito angloparlante. Me refiero a Juan José López-Ibor padre, quien en 1950 en su libro "La angustia vital" y luego más detalladamente en su libro “Las neurosis como enfermedades del ánimo”, del año 1966, planteó el concepto de "timopatía ansiosa". Su punto de partida fue el hecho de observación que muchos de los cuadros llamados por entonces "neurosis" tenían características que recordaban a las depresiones endógenas, como por ejemplo el ritmo horario, su vinculación al ritmo menstrual, a las estaciones del año, así como la frecuencia con que se presentaban en ellas las sustituciones de un síntoma por otro o de todo el síndrome por otro en apariencia diferente. Él observó además la tendencia a "encarnarse" de la angustia patológica, afirmando que mientras más se somatiza la angustia, más patológica es. “El enfermo nota una angustia patológica no sólo como un malestar, un dolor difuso ligado a la experiencia primaria de la corporalidad, sino anclado en determinados lugares de su cuerpo. La angustia anormal se concreta en la geografía somática (dolor epigástrico, opresión precordial, nudo en la garganta, 


\section{Los síndromes ansiosos y depresivos como timopatías}

cefalea, etc.) y también en la geografías psíquica: las fobias." (1966, p. 96). La angustia sería una experiencia límite que en algún momento se hace insoportable. En ese momento, cuando el paciente va a ser invadido por la misma nada, es cuando él se adhiere a algo que se presenta como un "motivo" de la angustia. Y ese sería el mecanismo de las génesis de las fobias. Estas tendrían un claro papel defensivo en la dinámica del proceso angustioso.

López-Ibor interpreta este síntoma dominante de los cuadros neuróticos, que es la angustia, como un sentimiento vital anormal, vital en el sentido de Kurt Schneider (1935) y de Max Scheler, muy cercano a la corporalidad y establece entonces el paralelismo con los cuadros depresivos. También en estos habría un compromiso de los sentimientos vitales, pero fundamentalmente del temple de ánimo. $\mathrm{Y}$ entonces propone hablar de estos cuadros que se encuentran igualmente anclados en la corporalidad, como la timopatía depresiva y la timopatía ansiosa. Ahora, nosotros proponemos englobar ambos tipos de síndromes, tanto los ansiosos como los depresivos, bajo la categoría superior de "timopatías", no en último término, porque investigaciones rigurosas de los últimos años no han podido separar, por ejemplo, la depresión mayor del trastorno generalizado de ansiedad. Así, Kessler y colaboradores, en el estudio sobre comorbilidad del Nacional Institute of Health, encontraron que el 72\% de los pacientes con TAG tenían también diagnóstico de depresión mayor y que, a la inversa, el 48\% de los pacientes diagnosticados con depresión mayor mostraban una comorbilidad con trastorno generalizado de ansiedad. Por último, habría que mencionar el reciente estudio evolutivo de 40 años (G. Rubio y J. J. López-Ibor, 2007) de más de 200 casos diagnosticados por LópezIbor Sr. como "timopatías" ansiosas y/o depresivas en los 50 y los 60. Estos autores encontraron un patrón evolutivo muy estable: fobia simple y ansiedad de separación en la infancia, trastorno de ansiedad generalizada y trastorno de pánico en la juventud y primera adultez, trastornos depresivos en la edad madura y por último, trastornos somatomorfos en edades más avanzadas. Valdría plantearse legítimamente la pregunta: ¿estos distintos síndromes 


\section{Otto Dörr Zegers}

descritos y en cierto modo reificados por la psiquiatría empirista actual son realmente entidades diferentes o se trata más bien de una misma alteración basal que se manifiesta de distintas formas según la edad de presentación?

\section{Las timopatías como constitutivas de la condición humana}

Hay dos razones que nos hacen pensar que tanto la angustia como la depresión han acompañado al hombre desde sus comienzos.

\section{La angustia como experiencia primordial}

Es un hecho de observación que el primer ruido que emiten los niños recién nacidos tiene los sonidos de la a y de la g, j o k, dependiendo del idioma, o más bien, de la forma cómo en ese ámbito idiomático es escuchado el sonido. Así, por ejemplo, en Chile lo oímos como "agú" y en España lo oyen como "ajó". López-Ibor hijo, en una reciente y notable investigación lingüística (2009), ha podido demostrar que las primeras palabras de que se tenga registro contienen la raíz "aj” o "anj”, de la cual deriva "angustia" (anjó en sánscrito), pero también "vida" (ankh en egipcio antiguo) y "principio" (argé en griego). No podemos entrar ahora en detalles sobre esta y otras investigaciones que apuntan a lo mismo. Por otra parte, los antropólogos han demostrado que la angustia cósmica fue una de las primeras emociones de la especie homo. Esta tiene que ver con la experiencia de mirar el firmamento y también con la conciencia de la muerte. Somos la única especie conocida que tiene conciencia de la muerte y por ende, historicidad. López-Ibor padre afirma que "la primera noche debió ser tremenda para el primer hombre". Y luego continúa: "Al salir el nuevo día y ver que era como el día anterior, se sintió tranquilo. La noche sigue siendo para muchos angustiados un trasunto de la nada." (1966, pp. 97 y 98). Ahora, el hombre primitivo se defendió de la angustia primordial a través de los rituales, que ponían de algún modo límite a lo infinito del tiempo y del 


\section{Los síndromes ansiosos y depresivos como timopatías}

espacio. También los diferentes dioses del politeísmo primitivo contribuyeron a morigerar la experiencia angustiosa derivada de la conciencia de la muerte.

\section{Creatividad y enfermedades del ánimo}

Desde Aristóteles se conoce el estrecho vínculo entre genialidad y melancolía. En las últimas décadas han aparecido una serie de trabajos con distintas metodologías que demuestran una y otra vez que la intuición de Aristóteles estaba en lo cierto. Hay estudios biográficos y genealógicos (A. Juda, 1949; C. Martindale, 1972; K. R. Jamison, 1993) de grandes poetas ingleses de los siglos XVIII y XIX, que demuestran sin lugar a dudas la altísima proporción entre ellos de enfermedades del ánimo severas. William Blake (1757-1827), Lord Byron (1788-1824), Lord Tennyson (1809-1892), William Wordsworth (17701850), Samuel Coleridge (1772-1834), Percy Shelly (1792-1822), John Keats (17951821), etc., todos ellos sufrieron de claros episodios maníaco-depresivos o, al menos, de depresiones recurrentes. En el mundo alemán el número tampoco es escaso. Pensemos en Heinrich Heine, Heinrich von Kleist, Franz Grillparzer, Robert Schumann, Friedrich Nietzsche, Rainer Maria Rilke, etc., etc.

Pero también hay estudios empíricos con personalidades eminentes que estaban vivas al momento de la investigación. En un conocido estudio de Nancy Andreasen (1987) sobre un grupo de 30 escritores invitados a un taller en la Universidad de Iowa, se pudo constatar que el 80\% de ellos sufría de alguna enfermedad del ánimo y el 43\%, de una enfermedad bipolar. En el grupo control la proporción era del 30\% y del 10\% respectivamente. Kay R. Jamison realizó un estudio parecido en Inglaterra. Ella estudió a 57 poetas, dramaturgos, novelistas y artistas británicos. El criterio de selección fue que hubieran recibido algún premio de los que se otorgan en Inglaterra (1993). El 38\% de ellos estaba en tratamiento por alguna enfermedad del ánimo, pero había diferencias entre un tipo de hombres eminentes y otros. Así, por ejemplo, los poetas fueron los que mostraron más alto porcentaje de enfermedad mental y los artistas plásticos, 


\section{Otto Dörr Zegers}

el menor. La enfermedad bipolar era mucho más frecuente en los poetas que en el resto.

Ahora, ¿qué tiene que ver este conocido vínculo entre genialidad y melancolía con nuestra hipótesis de que tanto las enfermedades del ánimo como los cuadros ansiosos son de alguna manera constitutivos de la condición humana? Pues bien, ocurre que hay consenso entre arqueólogos, etnólogos y antropólogos en el sentido de que nuestra especie, el homo sapiens, surgió junto con el lenguaje abstracto hace alrededor de cien mil años (Bickerton, 1990, 2005), siendo este incluso uno de los argumentos que hace pensar que la esquizofrenia, cuyo rasgo central es la perturbación del pensamiento y/o lenguaje, tenga que ver también con la condición humana. Ahora, ¿cómo se sabe que el lenguaje apareció en ese momento y no en otro? Sería muy largo detallar aquí la teoría del "out of Africa" y por qué se piensa que la mutación que permitió el lenguaje ocurrió en un solo momento y en un solo lugar. Pero lo importante es que se sabe que hay lenguaje allí donde hay simbolización. Y esta se ha conservado en los dibujos de las cavernas. Y la caverna pintada más antigua descubierta hasta ahora tiene poco más de noventa mil años. No conozco, por cierto, estas cavernas más antiguas, pero sí - y no es mucha gente la que tiene la experiencia, porque desde hace varias décadas están cerradas al público - he visitado las cuevas de Altamira, cuya antigüedad es no menor de veinte mil años. Y puedo dar fe que se trata de manifestaciones artísticas de la más alta calidad, donde el manejo de la forma, del color y de la metáfora es simplemente emocionante. Con esto quiero decir que la condición humana surge vinculada a la creatividad. Y como esta está, como vimos, esencialmente ligada a las enfermedades del ánimo, creo no errar demasiado al afirmar que esta condición ha acompañado al hombre desde el comienzo de los tiempos. A todo esto habría que agregar el nexo existente entre enfermedad bipolar y estructura biotipológica, concretamente el biotipo pícnico-endomorfo. Y no habría argumentos para pensar que este biotipo humano, con su característica personalidad, no ha existido desde siempre. 


\section{Los síndromes ansiosos y depresivos como timopatías}

\section{Las timopatías como perturbaciones de la Befindlichkeit (disposición afectiva o disposicionalidad), en el sentido de Heidegger}

Como dijimos en la introducción, Heidegger distingue dos formas cooriginarias de estar el Dasein en el mundo: la comprensibilidad, que se manifiesta a través del lenguaje y el temple de ánimo o disposición afectiva. Esta segunda forma, traducida acertadamente, en nuestra opinión, al italiano como "disposicionalidad", corresponde al modo de encontrarse cada persona en cada momento en su cuerpo y en el mundo. El Dasein, en cuanto ser-en-elmundo no sólo tiene de partida una cierta comprensión de una totalidad de significados, sino que siempre está dispuesto afectivamente de alguna manera. Dicho con otras palabras, las cosas además de estar provistas de un significado, por decirlo así teórico, poseen en todo momento una valencia emotiva. En rigor el descubrimiento del mundo descansa en la disposicionalidad. Y así, una intuición o un razonamiento puros no podrían jamás descubrir, por ejemplo, una atmósfera amenazadora. Esa atmósfera, mucho antes de ser interpretada y elaborada por nuestro intelecto, es sentida en nuestro cuerpo. El Dasein no es nunca un sujeto puro. Él no puede ser un espectador desinteresado de las cosas. Y así, el proyecto dentro del cual el mundo se le aparece al Dasein no es como el a priori kantiano, una apertura a la razón, sino siempre un proyecto templado y de alguna manera tendencioso. Comprendemos el mundo a través del lenguaje, pero lo sentimos con nuestro cuerpo o, más precisamente a través de nuestro modo de encontrarnos en él de alguna manera, vale decir de la disposicionalidad.

Pero ésta crea en nosotros también una situación que nos limita la libertad, puesto que no podemos cambiarla a voluntad, como sí podemos hacerlo con un interés intelectual o con una decisión. Así, no podemos decidir estar tristes o alegres, pero sí, estudiar una materia u otra, viajar a un lado o a otro. En todo caso el mundo se nos aparece siempre a la luz de una cierta 


\section{Otto Dörr Zegers}

disposición afectiva o emotiva: alegría, miedo, tedio, indiferencia, etc. La disposición afectiva representa entonces una tremenda paradoja puesto que por un lado es lo que uno tiene de más propio e individual, pero al mismo tiempo lo más cambiante y lo más ajeno. Así la disposicionalidad nos pone frente al hecho que nuestro modo de captar y comprender el mundo es algo cuyos fundamentos mismos se nos escapan. El Dasein es lanzado al mundo (geworfen) ante todo como esta experiencia originaria de su cuerpo que venimos describiendo, pero que en último término no le pertenece. Eso es lo que Heidegger llama facticidad o "estado de yecto" (Geworfenheit). Ahora, es justamente en la facticidad donde anida la experiencia primordial de la angustia. Escuchemos a Heidegger: “El ante-qué de la angustia es el estar-en-el-mundo en cuanto tal [...] Nada de lo que está a la mano o de lo que está ahí-dentro del mundo funciona como aquello ante lo cual la angustia se angustia [...] El mundo adquiere carácter de una total insignificancia. En la angustia no comparece nada determinado como amenazante..." (Ser y Tiempo, traducción de J. E. Rivera, p. 208). Es uno de los más grandes aportes de Heidegger al conocimiento del ser humano el haberle otorgado a la angustia el carácter de una experiencia primordial, tanto más cuanto que ella va a constituir el camino para superar la "caída" (Verfallenheit) hacia el mundo del "uno" o del "se". "El angustiarse abre originaria y directamente el mundo en cuanto mundo" nos dice Heidegger (op. cit., p. 209), pero al mismo tiempo “ella revela al Dasein el estar vuelto hacia el más propio poder-ser, es decir, revela su ser libre para la libertad de escogerse y tomarse a sí mismo entre las manos" (p. 210).

Aquí no puedo dejar de recordar uno de los más profundos versos del poeta austriaco Rainer Maria Rilke:

\footnotetext{
"Así como la naturaleza abandona a los seres al riesgo de su oscuro deseo y no protege a ninguno en especial ni en el suelo ni en las ramas, así, tampoco nosotros somos más queridos
} 


\section{Los síndromes ansiosos y depresivos como timopatías}

por el fundamento de nuestro ser. Él nos arriesga."

Y hacia el final del poema hace la impresionante afirmación:

“Lo que en definitiva nos cobija

es nuestro estar desamparados..."

Esta capacidad de la angustia de abrir el mundo desde la absoluta indefensión y desamparo lo explica Heidegger del siguiente modo: "Pero sólo en la angustia se da la posibilidad de una apertura privilegiada, porque ella aísla. Este aislamiento recupera al Dasein, sacándolo de su caída y le revela la propiedad o la impropiedad como posibilidad de su ser" (p. 212). La palabra alemana para propiedad es Eigentlichkeit pero esta se entiende mejor en castellano como "autenticidad". Esto implica que la vía para una existencia auténtica pasa a través de la experiencia de la angustia. Sin embargo, no hay que olvidar el otro significado de la palabra Eigentlichkeit, cual es propiedad, por cuanto él nos va a permitir entender que toda existencia sana y auténtica tiene un carácter "apropiativo" en el sentido de Pelegrina (2006) y pasar luego a una comprensión de las enfermedades mentales como procesos "desapropiativos".

Pero así como hay una angustia que podríamos llamar existencial, que nos descubre el mundo y nos salva de la posibilidad de perdernos en la inautenticidad de la existencia, existen otras formas de angustia que en lugar de abrir el mundo lo cierran y que en lugar de salvarnos nos pierden. $\mathrm{Y}$ esas son las que encontramos en la patología mental y particularmente en los cuadros ansiosos y depresivos.

Difícil imaginar entonces un ámbito de lo humano donde pueda caber mejor el mundo de los trastornos del ánimo y el de los llamados trastornos de ansiedad que en el de la disposicionalidad de Heidegger. Esa esfera en la que 


\section{Otto Dörr Zegers}

estamos siempre de alguna manera y que sin embargo, escapa a nuestra disponibilidad. El misterio de las llamadas enfermedades endógenas, tan propias del ser humano, tan irreductibles a la comprensión a través de esquemas teóricos precipitados, sean de corte psicologista o biologicista, empieza a develarse si lo miramos en la perspectiva de esta revolucionaria descripción de Heidegger. Es la comprensión originaria del mundo a través del lenguaje lo que se altera o fracasa en las esquizofrenias, que nosotros proponemos llamar, por eso, “logopatías”. Y es ese primordial estar en nuestro cuerpo y, a través de él, en el mundo lo que se congela o amenaza en la depresión o en la angustia, razón por la cual proponemos subsumir ambos tipos de enfermedades bajo el concepto de timopatías.

\section{Referencias}

American Psychiatric Association: Diagnostic and Statistical Manual of Mental Disorders. (2000) Fourth Edition. Text Revision. DSM-IV-TR ${ }^{\mathrm{TM}}$ Washington, D. C.: American Psychiatric Association.

Andreasen, N. (1987) "Creativity and mental illness: Prevalence rates in writers and their first-degree relatives". American Journal of Psychiatry 144: 12881292.

Baeyer W. von, Häfner, H., Kiksker, K. P.(1964) Psychiatrie der Verfolgten. BerlinGöttingen-Heidelberg: Springer Verlag.

Berner, P. (1983) „Wiener Forschungskriterien (Endogenomorph-Zyklothyme Achsensyndrome)". In: Diagnosekriterien für Schizophrene und Affektive Psychosen. (1983). Weltverband für Psychiatrie.

Bickerton, D.(1990) Language and species. Chicago: University of Chicago .

Bickerton, D.(2005) “Del protolenguaje al lenguaje”. En: La especiación del homo sapiens moderno. Madrid: Triacastela.

Binswanger, L.(1960) Melancholie und Manie. Pfullingen: Neske Verlag.

Bleuler, E.(1975) Lehrbuch der Psychiatrie (1916). 13. Auflage, neubearbeitet von Manfred Bleuler. Berlín-Heidelberg-New York: Springer Verlag.

Psicopatologia Fenomenológica Contemporânea, 2014, 3 (1), 1-22 


\section{Los síndromes ansiosos y depresivos como timopatías}

Cole, J., McGuffin, P. \& Farmer, A. E.(2008) "The classification of depression: are we still confused?" The British Journal of Psychiatry 192, 83-85.

Dörr, O., Enríquez, J., Jara, C.(1971) "Del análisis clínico-estadístico del síndrome depresivo a una comprensión del fenómeno de la depresividad en su contexto patogénico". Rev. Chil. Neuropsiquiat. 10, No 1:17-39.

Dörr, O.(1979) "Análisis fenomenológico de la depresividad en la melancolía y en la epilepsia". Actas Luso-Esp. Neurol. Psiquiatr. 7 (2 $2^{\text {a }}$ Etapa): 291-304.

Dörr, O., Tellenbach, H.(1980) "Differentialphänomenologie des depressiven Syndroms". Der Nervenarzt 51: 113-118.

Dörr, O.(1993) "Fenomenología de la corporalidad depresiva". Salud Mental 16: $22-$ 30.

Dörr, O.(2010) "Esquizofrenia, lenguage y evolución (o las esquizofrenias como logopatías)". Actas españolas de psiquiatría. 38(1). pp 1-7.

Farmer, A. \& McGuffin, P.(1989) "The Classification of the Depressions. Contemporary Confusion Revisited". British Journal of Psychiatry 155, 437-443.

Feighner, J. P., Robins, E., Guze, S. B., Woodruff, R. A., Winokur, G., Munoz, R.(1972) Diagnostic Criteria for Use in Psychiatric Research. Arch. Gen. Psychiatry 26: 57-63.

Heidegger, M.(1997) Ser y Tiempo. Traducción, prólogo y notas de J. E. Rivera. Santiago: Editorial Universitaria.

Jamison, K. R.(1993) Touched with Fire: Manic-Depressive Illness and the Artistic Temperament. New York: The Free Press, Division of Macmillan, Inc.

Jaspers, K.(1959) Allgemeine Psychopathologie. 7. Auflage. Berlin-GöttingenHeidelberg: Springer Verlag.

Juda, A.(1949) "The relationship between highest mental capacity and psychic abnormalities". American Journal of Psychiatry 106: 296-307.

Kessler, R. C., Nelson, C. B., McGonagle, K. A., Liu, J., Schwartz, M., Blazer, D. G.(1996) Comorbidity of DSM III R Major Depressive Disorder in the General Population: Results from US National Comorbidity Survey. Br. J. Psychiatric 168 Suppl. 30): 17-30.

Psicopatologia Fenomenológica Contemporânea, 2014, 3 (1), 1-22 


\section{Otto Dörr Zegers}

Kraepelin, E.(1916) Einführung in die Psychiatry. 3. Auflage. Leipzig: Johann Ambrosius Barth.

López Ibor Sr., J. J.(1966) Las neurosis como enfermedades del ánimo. Madrid: Editorial Gredos, S. A.

López-Ibor Jr., J. J., López-Ibor, M.-I.(2009) “Anxiety and logos: Toward a linguistic analysis of the origin of human thinking". J. Affect. Disord. XXX: 1-11.

Martindale, C.(1972) "Father's absence, psychopathology and poetic eminence". Psychological Reports 31: 843-847.

Parker, G.(2005) “Beyond major depression”. Psychological Medicine 35, 467-474.

Parker, G.(2006) “Through a Glass Darkly: The Disutility of the DSM Nosology of Depressive Disorders". Can J Psychiatry, Vol 51, No 14, December: 879886.

Pelegrina, H.(2006) Fundamentos Antropológicos de la Psicopatología. Madrid: Ediciones Polifemo.

Pfeiffer, W. M.(1969) "Die Symptomatik der Depression in transkultureller Sicht". En: Das depressive Syndrom. Hanns Hippius und Helmuth Selbach (Hrsg.). München-Berlin-Wien: Urban \& Schwarzenberg.

Pfeiffer, W. M.(1971) Transkulturelle Psychiatrie. Ergebnisse und Probleme. Stuttgart: Georg Thieme Verlag.

Rilke, R. M.(1956) „Widmung für Helmuth Freiherrn Lucius von Stoedten“. In: Sämtliche Werke, Band II. Frankfurt am Main: Insel Verlag.

Rubio, G., López-Ibor, J. J.(2007) “Generalized anxiety disorder: a 40-year follow-up study". Actas Psiquiat Scand 115: 372-379.

Rubio, G., López-Ibor, J. J.(2007) What can be learnt from the natural history of anxiety disorders? Eur Psychiatry 22: 80-86.

Scheler, M.(1948) Wesen und Formen der Sympathie. Frankfurt am Main: Schulte Verlag.

Schneider, K.(1935) Pathopsychologie der Gefühle und Triebe. Leipzig: Georg Thieme Verlag.

Schwartz, M. A., Wiggins, O. P.(1985) „Typifications. The First Step for Clinical Diagnosis". The Journal of Nervous and Mental Disease 175: 65-77. 


\section{Los síndromes ansiosos y depresivos como timopatías}

Schwartz, M. A., Wiggins, O. P.(1987) „Diagnosis and ideal types: a contribution to psychiatric classification". Comprehensive Psychiatry 28: 2771-91.

Spitzer, R. L., Endicott, J., Robins, E.(1982) Forschungs-Diagnose Kriterien (RDC). Traducido al alemán por H. E. Klein. Weinheim \& Basel: Beltz Verlag. 